\title{
Electron Acceleration by Self-Modulated Laser Wakefield in A Relativistically Self-Guided Channel
}

\author{
S.-Y. Chen, R.Wagner, A. Maksimchuk, D. Umstadter \\ Center for Ultrafast Optical Science, University of Michigan, Ann Arbor, MI \\ 48109
}

\begin{abstract}
The relativistic self-focusing of an intense laser pulse $\left(\mathrm{I} \sim 4 \times 10^{18} \mathrm{~W} / \mathrm{cm}^{2}, \lambda\right.$ $=1 \mu \mathrm{m}, \tau=400 \mathrm{fs}$ ) in a gas jet $750 \mu \mathrm{m}$ in length was observed using sidescattering imaging. A self-modulated laser wakefield was generated to accelerate self-trapped electrons. The energy distribution and transverse emittance of the electron beam changed due to the onset of the relativistic self-guiding.
\end{abstract}

\section{Introduction}

Plasma-based accelerators are of great interests for electron acceleration $[1,2]$ because they have the ability to support much larger acceleration gradient $(\mathrm{E}$ $>100 \mathrm{GV} / \mathrm{m}$ ) than convenctional RF accelerators. Several methods can be used to drive large amplitude plasma wave. Since the size of terawatt laser systems has been reduced by chirped pulse amplification, the laser wakefield accelerator (LWFA) [1] and the self-modulated LWFA [1,3-9], have recently received considerable attention and show promising results. In order to drive a plasma wave to large amplitudes, the laser pulse has to be focused to small spot size (on the order of tens of microns) to achieve high intensity. Nonetheless, it also limits the length of the plasma wave channel to be less than twice the Rayleigh length (confocal parameter) due to the laser beam diffraction. In order to extend this plasma wave channel, two methods have been proposed. One way is to use a prepulse to create a concave plasma channel which can then guide the main laser pulse over the length of this channel [3,10-11]. Another way is to make the laser pulse self-guided by relativistic self-focusing (self-guiding) so that it can propagate over many Rayleigh lengths while maintaining high peak intensity [12-14]. In this paper we, for the first time, report the interplay between relativistic self-guiding and acceleration of electrons in self-modulated laser wakefield.

\section{Experiments}

When a terawatt-peak-power laser is focused to high intensity into a gas , a plasma is created by tunneling ionization, and the free electrons begin to quiver at velocities close to the speed of light $(c)$ in the laser's transverse oscillating 
electromagnetic field. The laser light group velocity $v_{\mathrm{g}}$ depends on the index of refraction $v_{\mathrm{g}}=c n$, where the latter is given by

$$
n=\left[1-\left(\omega_{p} / \omega\right)^{2}\right]^{1 / 2},
$$

in which $\omega$ is the laser frequency and the plasma frequency is given by

$$
\omega_{p}=\left(4 \pi n_{e} e^{2} / m_{0}\right)^{1 / 2}
$$

where $e$ is the electron charge, $m_{0}$ is the electron rest mass, and $n_{e}$ is the plasma electron density. The relativistic factor $\gamma$ associated with the motion perpendicular to the direction of laser propagation depends on the laser field through the electron quiver velocity $v_{\mathrm{os}}, \gamma=\left[1+a^{2}\right]^{1 / 2}$, where $a=\gamma v_{\mathrm{os}} / c=e E / m_{0} \omega c=8.5 \times 10^{-}$ ${ }^{10} \lambda[\mu \mathrm{m}] I^{1 / 2}\left[\mathrm{~W} / \mathrm{cm}^{2}\right]$ is the normalized vector potential, $E$ is the laser electric field, and $I=c E^{2} / 8 \pi$ is the laser intensity. For a terawatt laser pulse, with $\gamma>1$, several nonlinear effects arise. First, the plasma frequency decreases when the laser intensity increases, due to the increase in $\gamma$. Second, the rapid temporal change in $\gamma$ for a short laser pulse broadens and modulates the transmitted light spectrum (relativistic self-phase modulation). Finally, the relativistic self-focusing effect, which occurs because the laser intensity varies transversely, can focus the laser to a smaller spot size. This increases the peak laser intensity and extends the length of the high intensity region, forming a channel. Usually, when self-focusing occurs, electron cavitation, which is the expelling of electrons out of laser axis by the transverse ponderomotive force, will also occur, enhancing the self-guiding of the laser beam $[12,15]$ and lowering the electron density. Theoretical work shows that this self-focusing (self-guiding) occurs at a critical power given approximately by $P_{\mathrm{c}}=17\left(\omega / \omega_{\mathrm{p} 0}\right)^{2} \mathrm{GW}$, where $\omega_{\mathrm{p} 0}$ is $\omega_{\mathrm{p}}$ in eqn.2 with $\gamma=1$ [16].

Laser pressure (ponderomotive force)-combined with ion inertia, which provide an electrostatic restoring force-can drive a high amplitude electron plasma wave (epw). By this process, some of the laser energy is converted to a longitudinal electrostatic laser wakefield, propagating at near the speed of light, which can continuously accelerate electrons in the direction of laser propagation. If the laser pulse width $\tau$ is much larger than a plasma period, $\tau>>2 \pi / \omega_{p}$, the resulting local density changes will have time to feedback on the light pulse. This case is referred to as the stimulated Raman forward scattering (RFS) instability [35]. Modulations in the index of refraction break the pulse into a sequence of shorter pulses with a periodicity equal to a plasma period, which resonantly drive the electron plasma wave to high amplitude. Once the plasma wave is excited, it can trap hot background electrons which may be heated by above-thresholdionization (ATI) [17] or Raman backscattering, sidescattering, and small angle 
forward scattering [18], and accelerate them to relativistic energies. The acceleration of trapped electrons will in turn damp the plasma wave, limiting the plasma wave amplitude and its temporal duration [19].

In the present experiments, a hybrid Ti:Sapphire - Nd:Glass laser system based on chirped pulse amplification and capable of delivering $3 \mathrm{~J}, 400 \mathrm{fs}$ laser pulses at $\lambda=1.053 \mu \mathrm{m}$ was used to drive the self-modulated LWFA. The $43 \mathrm{~mm}$ diameter beam was focused by an $\mathrm{f} / 4$ off-axis parabolic mirror to a spot size $\left(\mathrm{e}^{-2}\right.$ intensity) of $r_{0}=8.5 \mu \mathrm{m}$, giving a maximum vacuum intensity of $I=6.2 \times 10^{18}$ $\mathrm{W} / \mathrm{cm}^{2}\left(a_{0}=2.2\right)$. The laser was focused onto a supersonic helium gas jet with a sharp gradient $(250 \mu \mathrm{m})$ and a flat-top width of $750 \mu \mathrm{m}$. The neutral gas density varied linearly with backing pressure and reaches $1.8 \times 10^{19} \mathrm{~cm}^{-3}$ at a backing pressure of $1000 \mathrm{psi}$. An underdense plasma is created when the gas is tunnelingionized by the foot of the laser pulse.

The existence of a large amplitude epw is inferred from the presence of up to the 4th anti-Stokes sideband in the Raman forward scattering spectra obtained with a prism spectrometer [9]. In order to determine the absolute plasma wave amplitude and its temporal distribution, a copropagating green probe pulse generated by frequency-doubling $20 \%$ of the pump pulse is used to probe the plasma wave by using collective Thomson scattering [19]. The plasma wave amplitude is observed to be about $1.5 \mathrm{ps}$ in duration and the peak amplitude changes from $10 \%$ of $n_{0}$ to $40 \%$ of $n_{0}$ when the laser peak power is increased from $2 \mathrm{TW}$ to $3 \mathrm{TW}$, where $\mathrm{n}_{0}$ is the background electron density.

A sidescattering imaging system composed of collecting lens, filter, and $12-$ bit CCD is used to measure to spatial distribution of laser-plasma interaction. Fig. 1 shows side-scattering intensity distribution along the laser axis as a function of laser power for fixed gas density. At low laser power $(<1 \mathrm{TW})$, the channel length is fixed around $125 \mu \mathrm{m}$. When laser power exceeds $1.8 \mathrm{TW}\left(3.9 P_{\mathrm{c}}\right)$, the channel length extends to $250 \mu \mathrm{m}$ and stays fixed with increasing laser power. When the laser power is increased above $3.3 \mathrm{TW}\left(7.2 P_{\mathrm{c}}\right)$, the length extends futher to 750 $\mu \mathrm{m}$, which seems to be limited by the gas jet used in this experiment. The peaks of the images show the position of multi-foci, which seem to stay fixed in space regardless of laser power. Spectral analysis of the sidescattered light using imaging spectrometer shows that $>96 \%$ of the emission comes from the Thomson scattering of the blue-shifted pump light. Imaging of the Thomson scattering by using narrow bandpass filter centered at $\lambda$ shows an emission channel of the exactly same spatial distribution, only much weaker. 


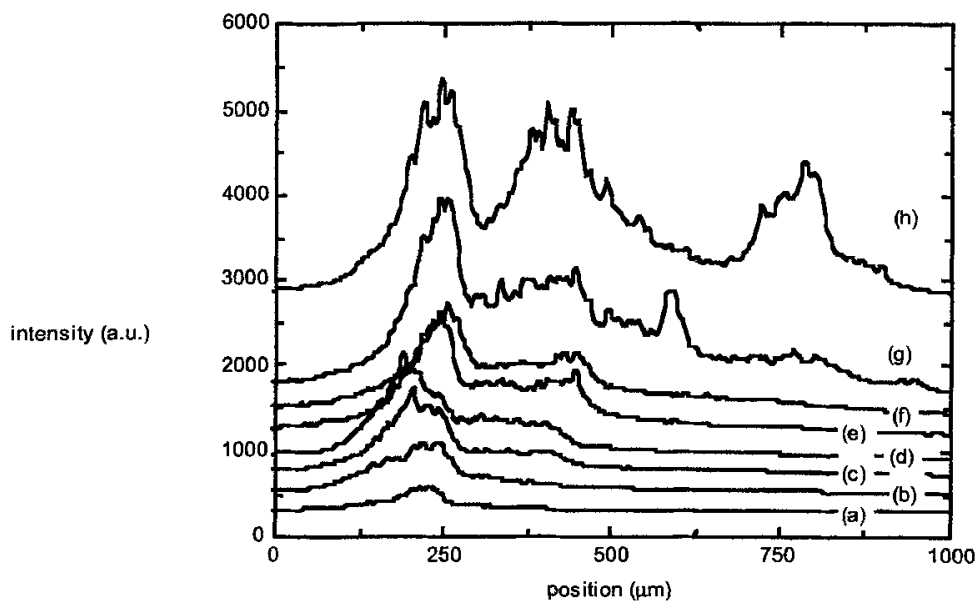

Fig. 1 On-axis lineouts of the sidescattering images as a function of peak laser power for fixed backing pressure 1000 psi (corresponding to a gas density of $1.8 \times 10 \mathrm{~cm}^{-3}$ ). (a) $0.8 \mathrm{TW}$ (b) $1.2 \mathrm{TW}$ (c) $1.5 \mathrm{TW}$ (d) $1.9 \mathrm{TW}$ (e) $2.5 \mathrm{TW}$ (f) $2.8 \mathrm{TW}$ (g) $3.4 \mathrm{TW}$ (h) $3.8 \mathrm{TW}$

A recent simulation and experiment [12] shows that when $P_{0} / P_{\mathrm{c}}=3-4$, a second focus is clearly visible (defined as the criterion for self-focusing), and when $P_{0} \approx 6.4 P_{\mathrm{c}}$, guiding occurs (appearance of four foci or a flat extended channel after two foci). The consistency between our result and theirs indicates that the extension of the plasma wave channel in this case is actually due to the relativistic self-guiding. A similar channel extension occurs if the gas density is varied at fixed laser power. For a $3.9 \mathrm{TW}$ laser pulse, the channel extends to $250 \mu \mathrm{m}$ at $400 \mathrm{psi}$ backing pressure $\left(3.2 P_{c}\right)$ and $750 \mu \mathrm{m}$ at $800 \mathrm{psi}\left(7.0 P_{c}\right)$. This consistent behavior at specific values of $P_{c}$ for varying laser power or plasma density indicates again that the channeling mechanism is relativistic self-focusing. Because of the ionization defocusing effect, the laser beam divergence increases by a factor of two with respect to the beam divergence in vacuum at all laser powers. The fact that the laser beam divergence doesn't increase when self-guiding occurs indicates that the cross section of the channel is close to the size without self-guiding.

The copropagating collective Thomson scattering is also used to monitor the change of plasma frequency when self-guiding occurs. As seen in Fig.2, the plasma wave frequency decreases gradually from $3.3 \times 10^{14} \mathrm{rad} / \mathrm{s}$ to $2.5 \times 10^{14} \mathrm{rad} / \mathrm{s}$ with increasing laser power. Three effects could cause the decrease of plasma wave frequency in this case. First, the plasma frequency decreases with increasing laser intensity, as can be seen in eqn.2. Second, the plasma wave frequency will 
decrease when the plasma wave amplitude grows larger and plasma wave becomes nonlinear. Third, when self-focusing occurs, the increase of laser intensity can expel the electrons out of the axis (electron cavitation), reducing the electron density and thus the plasma frequency. It is believed that the first mechanism is dominating here [21].

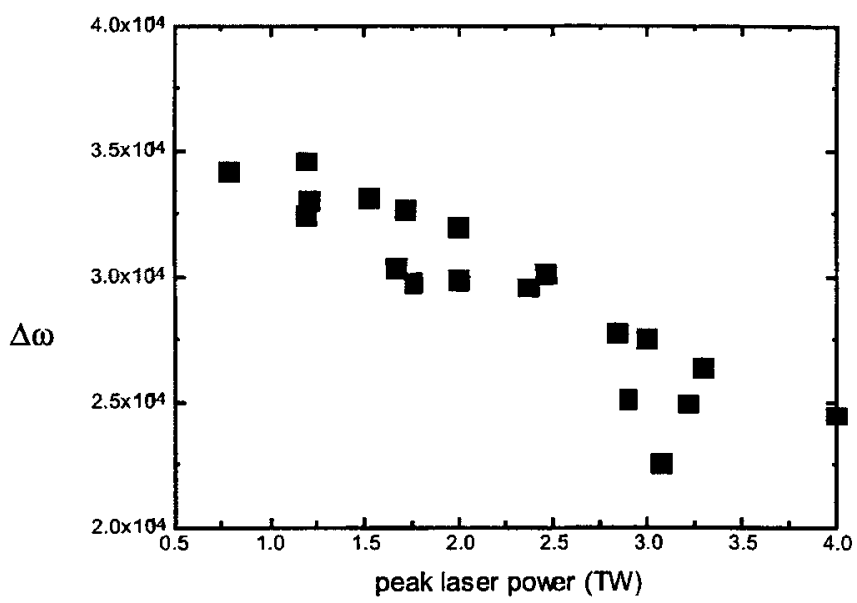

Fig. 2 Plasma wave frequency as a function of peak laser power.

The energy spectrum of the accelerated electron beam is measured by a collimator and a $60^{\circ}$ sector dipole magnet for low energy range $(<3 \mathrm{MeV})$, a rectangular dipole magnet for medium energy range $(2 \mathrm{MeV}-15 \mathrm{MeV})$ and aluminum absorbers for high energy range. The detector is a LANEX film (scintillating film) imaged by a CCD. The highest electron energy is known to be larger than $15 \mathrm{MeV}$, but the maximum value has not been determined yet. Fig.3 shows the electron kinetic energy distribution in the low energy range with varying laser power or plasma density. The number of electrons decreases exponentially with higher electron energy. In higher energy range, the electron number decreases much more slowly with higher electron energy (the slope decreases by a factor of 5 or more) compared to the low energy range. The slope of this energy distribution on the semilog scale basically decreases with higher laser power or higher gas density due to the fact that a larger plasma wave amplitude is achieved. In addition, a sudden jump in the slope is observed when 
self-guiding occurs by changing laser power or gas density. The extension of plasma channel not only provides longer acceleration distance but also allows the plasma wave to grow to larger amplitude. Recent PIC simulations show that such exponential distribution is typical for acceleration of self-trapped electrons and the slope of electron energy distribution in the low energy range becomes less steep with longer propagation distance [22]. In addition, the sudden jump of channel length should result in a sudden jump of electron energy distribution, as in our observation. About $50 \%$ of the accelerated electrons have kinetic energy higher than $1 \mathrm{MeV}$ and the energy transfered to the electron beam is about $0.5 \mathrm{~mJ}$.

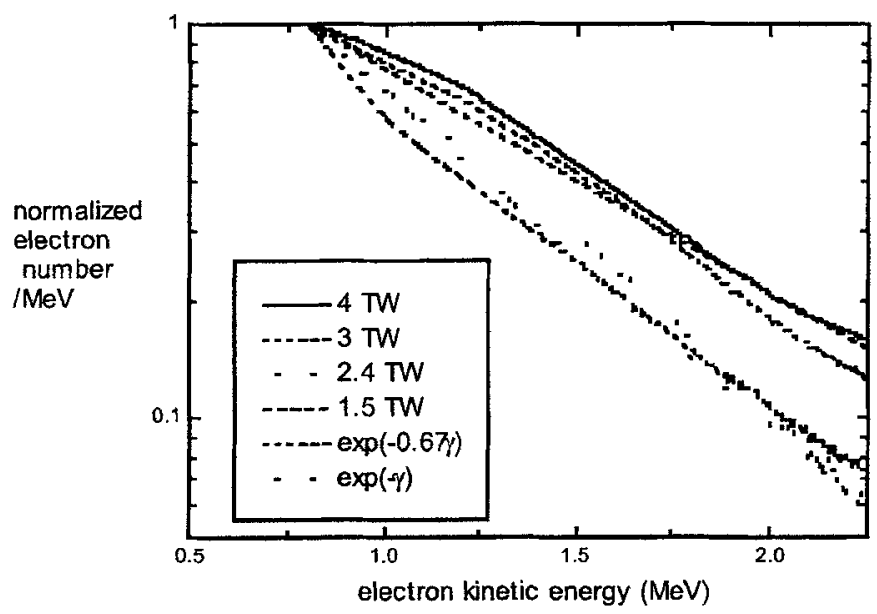

Fig. 3(a) Normalized electron energy distribution as a function of laser power for fixed backing pressure (1000 psi). Also shown are the fits to exponential function. 


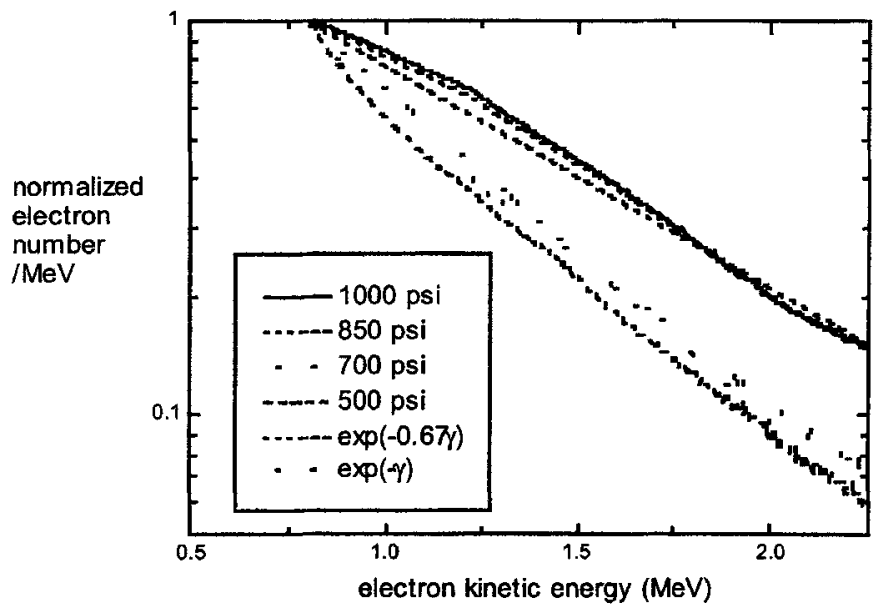

Fig. 3(b) Normalized electron energy distribution as a function of gas backing pressure (electron density) for fixed laser power (3 TW). Also shown are the fits to exponential function.

The number of accelerated electrons are measured using both a Faraday cup and a plastic scintillator coupled to a photo-multiplier tube. The electron acceleration occurs with a sharp threshold at $\sim 750 \mathrm{GW}(1.5 P \mathrm{c})$ and then the number increases exponentially with laser power and gradually saturates after 2 TW. At $3 \mathrm{TW}\left(6 P_{c}\right), 6 \times 10^{9}$ electrons are accelerated out of the plasma toward the forward direction (i.e., the laser propagation direction). Although the plasma wave should pick up a factor of three more electrons when self-guiding occurs (longer channel), it is difficult to observe this change since the electron number is increasing exponentially and the shot-to-shot fluctuation is large. 


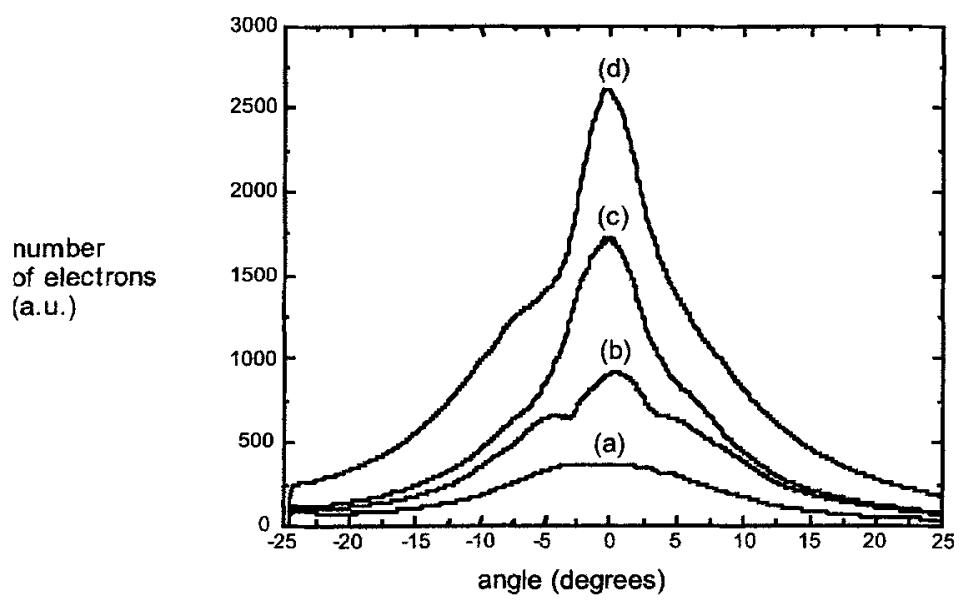

Fig. 4 Electron beam divergence relative to the laser propagation direction as a function of peak laser power. $\mathrm{P} / \mathrm{Pc}=$ (a) 3.4, (b) 5.0 , (c) 6.0, and (d) 7.5 .

The accelerated electrons are well confined in the forward direction since the accelerating field is in the forward direction and the transverse field can confine the beam transversely during acceleration.. Fig. 4 shows the angular distribution of accelerated electron beam with changing laser power. Because the number of electrons in the high energy range is very small compared to electrons at low energy $(<3 \mathrm{MeV})$, this image is basically the distribution of electrons in the range of $100 \mathrm{keV}-3 \mathrm{MeV}$, where $100 \mathrm{keV}$ is the energy electrons need in order to pass through the plastic and aluminum piece in front of the LANEX. Using aluminum absorbers, it is found that the electron beam divergence is independent of electron energy in this energy range. For the high energy range, it is expected that the beam divergence should decrease with higher electron energy. At low laser power, the electron beam divergence is measured to be about $10^{\circ}$ in radius at half maximum and the beam profile is Gaussian-like. When the laser power is increased and the channel length extends to $\sim 250 \mu \mathrm{m}$, a new distribution of smaller divergence starts to grow out of the original distribution. Above $2 \mathrm{TW}$, the electron beam divergence becomes $5^{\circ}$ in radius and the beam profile is Lorentzian-like. This minimum electron beam divergence possibly comes from the space charge effect after leaving the plasma wave, which can be large due to the fact that most of the electrons are in the few $\mathrm{MeV}$ range ( $\gamma$ small) and the current is high (large electron number and 
short electron bunch). Assuming $10^{9}$ electrons of $1 \mathrm{MeV}$ kinetic energy and $1 \mathrm{ps}$ bunch length, the electron beam divergence due to the space charge effect is calculated to be $6^{\circ}$. The decrease of electron beam divergence with laser power is caused by the increase of electron energy (lower space charge effect and relatively low transverse momemtum). The electron beam emittance defined by the radius of the source multiplied by the angle of beam divergence is $0.4 \pi$-mm-mrad in the best case and is same regardless of electron energy in the low energy range.

In summary, a collimated electron beam is produced by self-modulated laser wakefield, and the effect of relativistic self-focusing on electron acceleration was studied. The existence of relativistic self-guiding is observed by sidescattering imaging. The electron beam energy distribution and divergence changes when selfguiding occurs, leading to lower beam emittance and higher energy.

\section{Acknowledgements}

The authors would like to thank E. Dodd, D. Gustafson, G. Mourou, C. Keppel, and W. Buck for their support and useful discussions. This work was supported by DOE/LLNL subcontract B307953 and NSF STC PHY 8920108.

\section{References}

[1] T. Tajima and J. Dawson, Phys. Rev. Lett. 43, 267 (1979).

[2] E. Esarey et al., IEEE Trans. Plasma Science 24, 252 (1996).

[3] P. Sprangle et al., Phys. Rev. Lett. 69, 2200 (1992).

[4] T. M. Antonsen and P. Mora, Phys. Rev. Lett. 69, 2204 (1992).

[5] N. E. Andreev et al., JETP Lett. 55, 571 (1992).

[6] C. A. Coverdale et al., Phys. Rev. Lett. 74, 4659 (1995).

[7] K. Nakajima et al., Phys. Rev. Lett. 74, 4428 (1995).

[8] A. Modena et al., Nature (London) 377, 606 (1995).

[9] D. Umstadter et al., Science 273, 472 (1996).

[10] C. G. Durfee III et al., Phys. Rev. E 51, 2368 (1995).

[11] ] P. E. Young et al., Phys. Rev. Lett. 75, 1082 (1995).

[12] A. Chiron et al., Phys. Plasmas 3, 1373 (1996).

[13] P. Monot et al., Phys. Rev. Lett. 74, 2953 (1995).

[14] A. B. Borisov et al., J. Opt. Soc. Am. B 11, 1941 (1994).

[15] A. B. Borisov et al., Phy. Rev. A 45, 5830 (1992).

[16] C. Max et al., Phys. Rev. Lett. 33, 209 (1974).

[17] B. M. Penetrante et al., Phys. Rev. A 43, 3100 (1991).

[18] Y. Kishimoto et al., to be published.

[19] S. P. Le Blanc et al., to be published.

[20] S. C. Wilks et al., Phys. Plasmas 2, 274 (1995).

[21] A. Modena et al., IEEE Trans. Plasma Sci. 24, 289 (1996).

[22] W. B. Mori (private communication). 